\title{
DÜBLIN
}

Technological University Dublin

ARROW@TU Dublin

\section{A Competitive Random Sequential Adsorption Model for Immunoassay Activity}

\author{
Dana Mackey \\ Technological University Dublin, dana.mackey@tudublin.ie \\ Eilis Kelly \\ Technological University Dublin, eilis.kelly@tudublin.ie \\ Robert Nooney \\ Dublin City University
}

Follow this and additional works at: https://arrow.tudublin.ie/scschmatcon

Part of the Medical Biotechnology Commons, and the Other Applied Mathematics Commons

\section{Recommended Citation \\ Mackey, D., Kelly, E. \& Nooney, R. (2016) A Competitive Random Sequential Adsorption Model for Immunoassay Activity. The 19th European Conference on Mathematics for Industry, Santiago de Compostela, 13th.-17th. June 2016. doi:10.21427/cg7a-tb88}

This Conference Paper is brought to you for free and open access by the School of Mathematics at ARROW@TU Dublin. It has been accepted for inclusion in Conference papers by an authorized administrator of ARROW@TU Dublin. For more information, please contact arrow.admin@tudublin.ie, aisling.coyne@tudublin.ie,gerard.connolly@tudublin.ie. Funder: Irish Research Council 


\title{
A competitive random sequential adsorption model for immunoassay activity
}

\author{
Dana Mackey $^{1}$, Eilís Kelly ${ }^{1}$, Robert Nooney ${ }^{2}$ \\ ${ }^{1}$ School of Mathematical Sciences, Dublin Institute of Technology, Ireland, \\ dana.mackey@dit.ie \\ ${ }^{2}$ Biomedical Diagnostics Institute, Dublin City University, Ireland
}

\begin{abstract}
Immunoassays rely on highly specific reactions between antibodies and antigens and are used in biomedical diagnostics applications to detect biomarkers for a variety of diseases. Antibody immobilization to solid interfaces through random adsorption is a widely used technique but has the disadvantage of severely reducing the antigen binding activity and, consequently, the assay performance. This paper proposes a simple mathematical framework, based on the theory known as competitive random sequential adsorption (CRSA), for describing how the activity of immobilized antibodies depends on their orientation and packing density and generalizes a previous model by introducing the antibody aspect ratio as an additional parameter which could influence the assay behaviour.
\end{abstract}

\section{Introduction}

Antibodies and antigens are extensively used as biomarkers for a wide variety of immunodiagnostic applications such as pregnancy testing or cancer diagnostics. Many of these technologies rely on the immobilization of antibodies on solid support interfaces, through strategies such as physical adsorption or covalent cross-linking, which result in a random particle distribution and considerably reduced antigen-binding activity. This phenomenon, generally attributed to shielding of antibody active sites and molecule denaturation upon contact with the solid surface, has been widely researched and described in the experimental literature, [8], [11], [12]. Of additional interest is the relationship between antigen-capturing activity and antibody surface density, with many papers reporting that crowding of antibodies has a detrimental effect on assay signal, [8], [12], [10]. Therefore, one of the primary considerations in immunoassay design is optimizing the concentration of capture antibody in order to achieve maximal antigen binding and, subsequently, improved sensitivity and limit of detection.

Antibodies are large Y-shaped proteins composed of two regions: a fragment crystallizable $(\mathrm{Fc})$ region at the base (responsible for recruiting components of the 
immune system) and a fragment antigen binding (Fab) region at the top (see Figure 1). Each arm of the Fab region contains a hypervariable region at its tip, called a paratope, which is capable of binding strongly to one epitope on an antigen. The immobilized antibody orientation is usually described in the immunoassay literature (see, for example, [11], [12]) by one of the following positions: "end on" (the ideal position, with the active Fab region pointing towards the solution), "head on" (or Fab down), "flat on" and "side on" (both of which correspond, in our 1-dimensional model, to a side orientation, as shown in Figure 1).
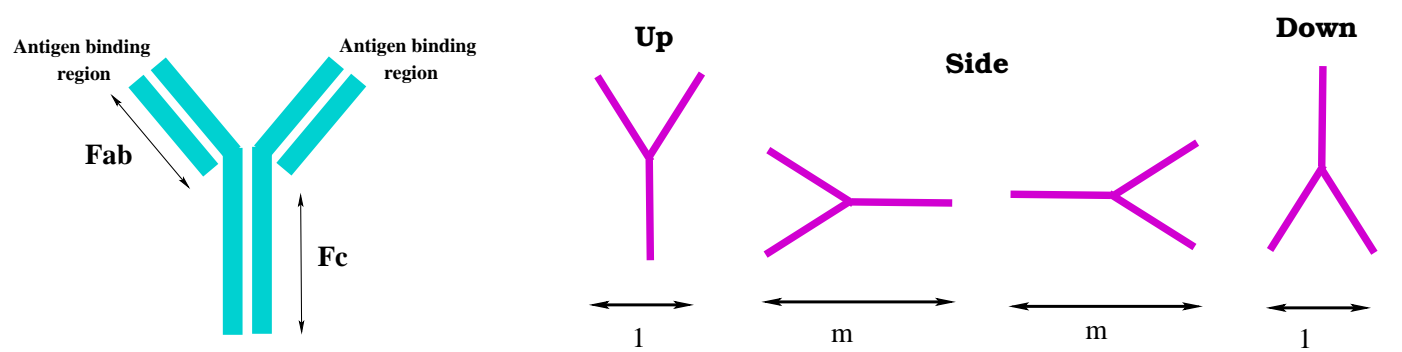

Figure 1: Antibody structure and immobilization orientations. Our model assumes that an immobilized particle covers a length of either 1 or $m$ on the 1-dimensional substrate.

The theory known as random sequential adsorption (RSA) has been successfully used over the past few decades to describe monolayer particle deposition, with wide applications in many physical and biological settings ([1], [4], [6]). In the standard description, uniform particles are transported to a surface at a constant rate and irreversibly deposited. The adsorption site is chosen randomly but, if the particle overlaps an already deposited one, it is rejected. The process is continued until the jamming limit is reached, that is no spaces are left on the substrate. In one dimension, this process is commonly referred to as "the car parking problem" (or interval filling) and the jamming coverage, also known as the Rényi constant, has been calculated in [5] to be $C_{R} \approx 0.74756$. A generalization known as competitive RSA (see, for example, [3]) deals with mixtures of particles having different sizes and adsorption probabilities.

We have recently presented a new mathematical model for quantifying the activity of antibodies immobilized on a solid surface, based on the RSA framework, [7]. In this model, the antibodies (or any other active molecules) were represented by oriented circles whose diameters cover a 1-dimensional substrate in the same manner as intervals fill a line in the standard car parking problem. A simple procedure was developed for estimating the percentage of antibodies which are correctly oriented (Fab site accessible to antigens in solution) out of total antibody coverage. Of particular interest is identifying parameter ranges for which the activity (and consequently the assay signal) increases with antibody concentration and then sharply decreases (the so-called "hook effect", see [8], [10]) which indicates the existence of a well-defined optimal surface coverage. 
The current paper generalizes our previous model to address the more realistic case where different immobilization configurations occupy intervals of different sizes in the above description. More specifically, the antibody width is assumed larger that its height and so the side-on and head-on orientations can be thought of as two competing adsorption strategies and described within the more general competitive RSA theory ([3]). The antibody aspect ratio is introduced in the analysis as another parameter which might influence the biosensor functionality.

\section{A model for estimating surface antibody activity}

The binary mixture RSA model described in [3] assumes that two particles of sizes 1 and $m(m>1)$ are selected with probabilities $q$ and $p=1-q$, respectively, to be deposited randomly on a one dimensional substrate of length $L$. The function $N(x, t)$ is defined to be the gap length density function at time $t$ (so $N(x, t) d x$ represents the mean number of gaps with length between $x$ and $x+d x)$ and we let $P(x, t)=$ $N(x, t) / L$. As $L \rightarrow \infty$, the time evolution of the gap size density is described by the following kinetic model

$$
\frac{\partial P(x, t)}{\partial t}= \begin{cases}-(x-\sigma) P(x, t)+2 q \int_{x+1}^{\infty} P(y, t) d y+2 p \int_{x+m}^{\infty} P(y, t) d y, & m<x<\infty \\ -q(x-1) P(x, t)+2 q \int_{x+1}^{\infty} P(y, t) d y+2 p \int_{x+m}^{\infty} P(y, t) d y, & 1<x \leq m \\ 2 q \int_{x+1}^{\infty} P(y, t) d y+2 p \int_{x+m}^{\infty} P(y, t) d y, & 0<x \leq 1,\end{cases}
$$

where the terms in all equations represent rates at which gaps of length $x$ can be created or destroyed, while $\sigma=m p+q$ denotes the average size of incoming molecules. (The kinetic equations for $P(x, t)$ in the single particle case were described in [4].) Using the initial conditions $P(x, 0)=0$ and $\lim _{t \rightarrow 0} \int_{0}^{\infty} x P(x, t) d x=1$ an analytical solution can be found, provided $1 \leq m \leq 2$, as follows:

$$
P(x, t)= \begin{cases}t^{2} F(t) e^{-(x-\sigma) t}, & m<x<\infty \\ 2 e^{-q(x-1) t} \int_{0}^{t} F(s) s\left[q e^{(m p-1) s}+p e^{-q m s}\right] e^{-x p s} d s, & 1<x \leq m \\ 2 \int_{0}^{t} s F(s) e^{\sigma s}\left[q e^{-(x+1) s}+p e^{-(x+m) s}\right] d s, & m-1<x \leq 1 \\ 4 q \int_{0}^{t} e^{q u} \int_{0}^{u} s F(s) \frac{q e^{(m p-1) s}+p e^{-q m s}}{q u+p s} \times & \\ \quad \times\left[e^{-(q u+p s)(x+1)}-e^{-(q u+p s) m}\right] d s d u & \\ \quad+2 \int_{0}^{t} u F(u)\left[q e^{(\sigma-m) u}+p e^{(\sigma-x-m) u}\right] d u, & 0<x \leq m-1\end{cases}
$$

where

$$
F(t)=\exp \left[-2 \int_{0}^{t} \frac{q\left(1-e^{-u}\right)+p\left(1-e^{-m u}\right)}{u} d u\right]
$$


The total coverage, $\theta_{T}(t)=\theta_{S}(t)+\theta_{L}(t)$, is comprised of the coverage by small particles, $\theta_{S}(t)$, and that of the large particles, $\theta_{L}(t)$, which are calculated in [3] as

$$
\begin{aligned}
\theta_{S}(t)= & 2 \int_{0}^{t} s F(s) e^{-\sigma s}\left[p+q e^{(m-1) s}\right] \times\left[\frac{e^{-(m-1)(p s+q t)}-1}{p s+q t}-\frac{e^{-(m-1) s}-1}{s}\right] d s \\
& +q \int_{0}^{t} F(s)[1+(m-1) s] e^{-(m-1) q s} d s \\
\theta_{L}(t)= & p m \int_{0}^{t} F(s) e^{-(m-1) q s} d s .
\end{aligned}
$$

Note that the jamming coverage $\theta_{T}(\infty)$ in the binary case (which depends on $m$ and $q$ ) exceeds the Renyi number for the one particle RSA theory and satisfies

$$
0.7475 . .=C_{R} \leq \theta_{T}(\infty) \leq C_{R}+C_{R}\left(1-C_{R}\right)=0.937 \ldots
$$

where the extreme values are obtained for $q=0$ and $m \rightarrow \infty, q \rightarrow 0$, respectively.

We assume for simplicity that, in our 1-dimensional description, antibodies can only adsorb in one of the four positions shown in Figure 1 (Fab site pointing up, down, left or right) and can only cover two possible lengths on the substrate (1 or $m$ ). We also assume that the probability of an up/down orientation (corresponding to the short side) is $q$ while the probability of a left/right (long side) adsorption is $p=1-q$. Similar to the model presented in [7], a particle is defined to be active if, either its binding site (Fab region) is pointing up, or else pointing left or right and a gap of length at least $\delta$ exists between the binding site and the neighbouring adsorbed molecule, where $\delta \leq 1$ depends on the size of the oncoming reactant molecules.

We now write the gap density function as

$$
P(x, t)=P_{L}(x, t)+P_{S}(x, t),
$$

where $P_{L}$ and $P_{S}$ denote the density of gaps with a large particle (or small particle, respectively) on the left. We similarly expand $P_{L}$ as

$$
P_{L}(x, t)=P_{L L}(x, t)+P_{L S}(x, t),
$$

where $P_{L L}, P_{L S}$ denote the gaps with a large particle on the left and a large (or small, respectively) particle on the right. The total number of active molecules at any given time, $N_{\text {active }}$, is then calculated by adding all "up" particles, all "left" particles with enough space on their left (which is obtained by multiplying the percentage of particles pointing left by the total number of gaps $\geq \delta$ ) and all "right" particles with enough space on their right (obtained in the same way as the left case). The "down" particles are assumed inactive. Moreover, if two adjacent particles are pointing towards the gap between them and this gap is not large enough to fit two antigens then only one of the immobilized particles is considered active. We can then write

$$
\frac{N_{\text {active }}}{L}=\frac{1}{2} \theta_{S}+\int_{\delta}^{\infty} P_{L}(x, t) d x-\frac{1}{4} \int_{\delta}^{2 \delta} P_{L L}(x, t) d x .
$$


The density $P_{L}$ can be calculated using the same approach as that described in the previous section for $P(x, t)$. For example, when $x>m$, this function satisfies the integro-differential equation

$\frac{\partial P_{L}}{\partial t}=-(x-\sigma) P_{L}(x, t)+q \int_{x+1}^{\infty} P_{L}(y, t) d y+p \int_{x+m}^{\infty} P_{L}(y, t) d y+p t F(t) e^{-(x+m-\sigma) t}$,

with similar equations for other $x$ ranges. This can be solved to give

$$
P_{L}(x, t)= \begin{cases}t F_{L}(t) e^{-(x-\sigma) t}, & m<x<\infty, \\ e^{-q(x-1) t} \int_{0}^{t} G(m-1, s) e^{-(x p+q m) s} d s, & 1<x \leq m, \\ \int_{0}^{t} e^{-(x+m-\sigma) s} G(m-1, s) d s, & m-1<x \leq 1, \\ q \int_{0}^{t} e^{-(m-1) q u} \int_{0}^{u} \frac{e^{-m s}}{q u+p s} G(m-1, s) \times & \\ \quad \times\left[e^{-(x-m+1)(q u+p s)}-1\right] d s d u & \\ \quad+\int_{0}^{t} G(x, u) e^{-(x+m-\sigma) u} d u, & 0<x \leq m-1,\end{cases}
$$

where

$$
F_{L}(t)=p F^{1 / 2}(t) \int_{0}^{t} F^{1 / 2}(s) e^{-m s} d s, \quad G(z, t)=q F_{L}(t) e^{z t}+p F_{L}(t)+p t F(t)
$$

and $F(t)$ is defined in (3). This procedure is repeated once more and, finally, we find

$$
P_{L L}(x, t)= \begin{cases}2 p e^{-(x-\sigma) t} \int_{0}^{t} F_{L}(s) e^{-m s} d s, & m<x<\infty, \\ 2 p e^{-q(x-1) t} \int_{0}^{t} F_{L}(s) e^{-s(p x+q m)} d s & 1<x \leq m, \\ 2 p \int_{0}^{t} F_{L}(s) e^{(\sigma-x-m) s} d s, & 0<x \leq 1 .\end{cases}
$$

The derivation of $N_{\text {active }}$ is greatly simplified if we assume that the gap distribution is independent of the particle size and hence the densities $P_{L}$ and $P_{S}$ are proportional to the number ratios of large and small particles, that is

$$
P_{L}(x, t)=\alpha_{L}(t) P(x, t), \quad P_{s}(x, t)=\alpha_{S}(t) P(x, t),
$$

where

$$
\alpha_{L}(t)=\frac{\theta_{L}(t)}{\theta_{L}(t)+m \theta_{S}(t)}, \quad \alpha_{S}(t)=\frac{m \theta_{S}(t)}{\theta_{L}(t)+m \theta_{S}(t)} .
$$

With this assumption, the number of active particles can be calculated as

$$
\frac{N_{\text {active }}}{L}=\frac{1}{2} \theta_{S}+\alpha_{L} \int_{\delta}^{\infty} P(x, t) d x-\frac{1}{4} \alpha_{L}^{2} \int_{\delta}^{2 \delta} P(x, t) d x
$$

which only uses the total gap distribution function $P(x, t)$ given in (2). 


\section{$3 \quad$ Results and conclusions}

The number of active particles is evaluated from (5) or (8), using the previously calculated density functions $P_{L}(x, t), P_{L L}(x, t)$ or $P(x, t)$. (Note that the two approaches to calculating $N_{\text {active }}$ give identical results.) The active particle concentration is then plotted as a function of the total concentration of immobilized antibodies, given by $\theta_{S}+\frac{\theta_{L}}{m}$, in Figure 2. We consider the cases of large antigen particles $(\delta=1$, so the antigen size is equal to that of the antibody) and small antigen particles $(\delta=0.1)$, each of these relevant to various biosensing applications. As expected, the antibody activity is consistently higher for small antigens, due to improved lateral accessibility (for side-lying particles). Recall that the total antibody coverage (and, hence, concentration), calculated as $\theta_{T}=\theta_{S}+\theta_{L}$ from (4), varies with the parameters $m$ (the aspect ratio) and $q$ (the probability of upright orientation).

The two groups of curves in each figure correspond to $q=0.1$ (side orientation prevails) and $q=0.8$ (most antibodies adsorb in the up/down positions). Within each group, the three curves (distinguished by different line styles) correspond to $m=1$, $m=1.5$ and $m=2$. (The case $m=1$ has already been discussed in [7]). The side orientation is generally associated with the hook effect (high activity and antigenbinding rate at low antibody coverage, which then decrease at higher concentrations), which is consistent with results reported in the experimental literature, [8], [12], [13], and explained by increased steric hindrance to antigen access.
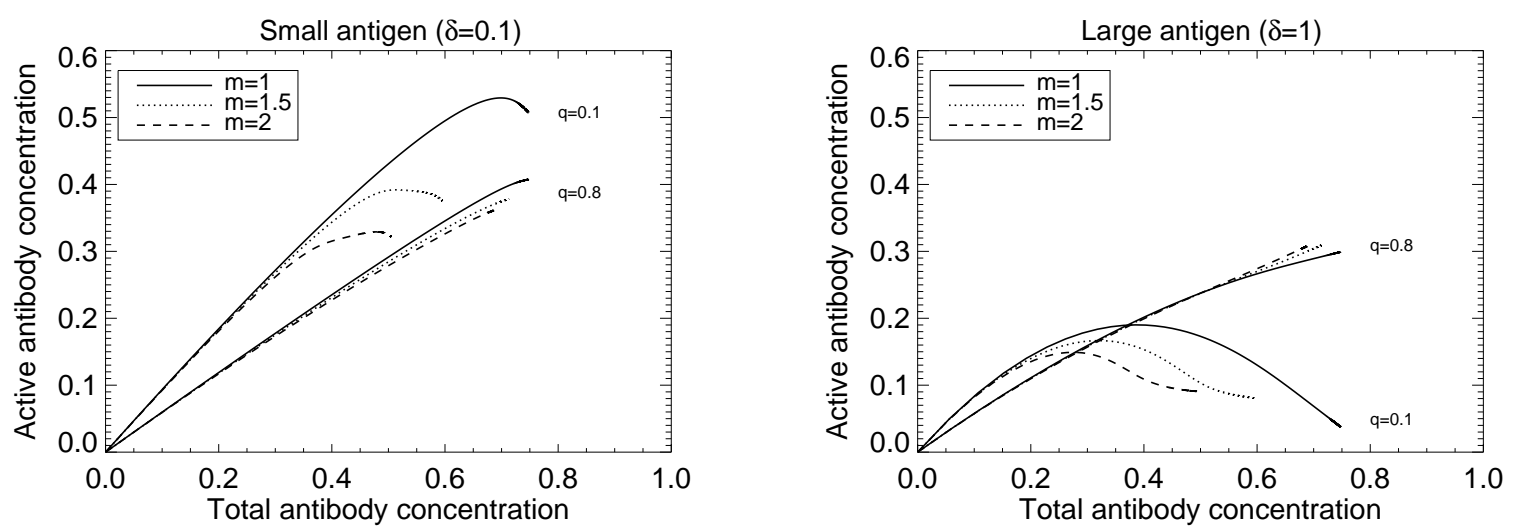

Figure 2: Active antibody concentration, $N_{\text {active }} / L$, as a function of total antibody concentration, $\theta_{S}+\left(\theta_{T} / m\right)$, for small $(\delta=0.1)$ and large $(\delta=1)$ antigen particles.

The aim of the current paper was to assess the effect of the antibody aspect ratio, $m$, on the immunoassay behaviour. It can be seen from Figure 2 that $m$ strongly influences activity when the side orientation predominates but has relatively little importance when antibodies are adsorbed in the up/down configurations. In the latter case, it would be acceptable to approximate the particle aspect ratio by $m=1$ and use our much simpler model in [7] to simulate the immobilized antibody activity. 
By contrast, when $q$ is small, it is seen that higher values of $m$ correspond to lower activity and so "circular" particles seem to be the most efficient.

As discussed in [7], many qualitative features of this modelling framework reproduce immunoassay properties reported in the experimental literature and support the conclusion of [9] that optimal performance is determined by the interplay of factors such as immobilized antibody density, relative size of antigens and method of immobilization. Therefore, a theoretical study of the relationship between these parameters should lead to improved design and functionality of such devices.

\section{Acknowledgement}

The second author gratefully acknowledges financial support from the Irish Research Council.

\section{References}

[1] Evans, J.W.: Random and cooperative sequential adsorption. Rev. Mod. Phys., $651281(1993)$

[2] Gubala, V., Crean, C., Nooney, R., Hearty, S., McDonnell, B., Heydon, K., O'Kennedy, R., MacCraith, B.D., Williams, D.E.: Kinetics of immunoassays with particles as labels: effect of antibody coupling using dendrimers as linkers. Analyst. 136, 2533-2541 (2011)

[3] Hassan, M.K., Schmidt, J., Blasius, B., Kurths, J.: Jamming coverage in competitive random sequential adsorption of a binary mixture. Phys. Rev. E. 65, (2002)

[4] Krapivsky, P.L.: Kinetics of random sequential parking on a line. J. Stat. Phys. 69, 135-150 (1992)

[5] Rényi, A.: On a one-dimensional problem concerning random space-filling (In Hungarian). Publ. Math. Inst. Hung. Acad. Sci, 3, 109-127 (1958)

[6] Roach, J.C., Thorsson, V., Siegel, A.F.: Parking strategies for genome sequencing. Genome Research, 10, 1020-1030 (2000)

[7] Mackey, D., Kelly, E., Nooney, R.: Modelling random antibody adsorption and immunoassay activity. Math. Biosci. Eng. 13, 6, 1159-1168, (2016)

[8] Saha, B. Evers, T.H., Prins, M.W.J.: How antibody surface coverage on nanoparticles determines the activity and kinetics of antigen capturing for biosensing. Anal. Chem., 86, 8158-8166 (2014)

[9] Schramm, W., Paek, S.: Antibody-antigen complex formation with immobilized immunoglobulins. Anal. Biochem., 205, 47-56 (1992) 
[10] Vareiro, M.L.M., Liu, J., Knoll, W., Zak, K., Williams, D., Toby, A., Jenkins, A.: Surface plasmon fluorescence measurements of human chorionic gonadotrophin: role of antibody orientation in obtaining enhanced sensitivity and limit of detection. Anal. Chem., 77, 2426-2431 (2005)

[11] Wiseman, M.E., Frank, C.W.: Antibody adsorption and orientation on hydrophobic surfaces. Langmuir, 28, 1765-1774 (2012)

[12] Xu, H., Lu, J.R., Williams, D.E.: Effect of surface packing density of interfacially adsorbed monoclonal antibody on the binding of hormonal antigen human chorionic gonadotrophin. J. Phys. Chem. B, 110, 1907-1914 (2006)

[13] Zhao, X., Pan, F., Cowsill, B., Lu, J.R., Garcia-Gancedo, L., Flewitt, A.J., Ashley, G.M., Luo, J.: Interfacial immobilization of monoclonal antibody and detection of human prostate-specific antigen. Langmuir, 27, 7654-7662 (2011) 